\title{
A single neurotoxic dose of MDMA decreases BDNF expression in the frontoparietal cortex but not in the hippocampus Szilvia Vas ${ }^{1}$, Diána Kostyalik¹ ${ }^{1}$ Csaba Ádori ${ }^{1}$, Rómeó D Andó ${ }^{2}$ and György Bagdy*1
}

\author{
Address: ${ }^{1}$ Institute of Pharmacodynamics, Semmelweis University, 1089 Budapest, Hungary and ${ }^{2}$ Institute of Experimental Medicine, Hungarian \\ Academy of Sciences, 1450 Budapest, Hungary \\ Email: György Bagdy* - bag13638@iif.hu \\ * Corresponding author
}

from 15th Scientific Symposium of the Austrian Pharmacological Society (APHAR) Joint meeting with the Hungarian Society of Experimental and Clinical Pharmacology (MFT) and the Slovenian Pharmacological Society (SDF)

Graz, Austria. 19-21 November 2009

Published: 12 November 2009

BMC Pharmacology 2009, 9(Suppl 2):A4I doi:I0.|I86/I47|-22I0-9-S2-A4 I

This abstract is available from: http://www.biomedcentral.com/I47I-22 I0/9/S2/A4I

(c) 2009 Vas et al; licensee BioMed Central Ltd.

\section{Background}

The popular recreational abuse drug 3,4-methylenedioxymethamphetamine (MDMA, 'Ecstasy') produces acute and long-lasting deficits in several markers of the serotonergic (5-HT) system. BDNF (brain derived neurotrophic factor) is a prominent trophic factor of serotonergic fibers. The aim of this study was to characterize the damage of serotonergic fibers in the frontoparietal cortex and hippocampus in parallel with the expression of BDNF.

\section{Methods}

Male Dark Agouti rats ( 7 weeks old) were treated with a single (15 mg/kg, i.p.) dose of MDMA. Density of tryptophan hydroxylase $(\mathrm{TpH})$ immunoreactive (ir) fibers was quantified 3 days and 3 weeks after treatment. BDNF protein and mRNA levels were measured by ELISA and quantitative PCR method 24 hours and 3 weeks after treatment. the quantitative PCR method. In contrast, no such a reduction was found in the hippocampus.

\section{Conclusion}

Our findings show that administration of a single dose of MDMA, a neurotoxic agent, leads to a significant drop in the TpH-ir axon density with a parallel decrease in BDNF levels 3 days and 3 weeks after treatment in the frontoparietal cortex. In contrast, in the hippocampus, the reduction of TpH-ir axon density is not accompanied by the decrease of BDNF level. These results highlight regional differences in the expression of BDNF, the main neurotrophic factor of serotonergic fibers, in the terminal serotonergic areas after a neurotoxic dose of MDMA.

\section{Acknowledgements}

Supported by the EC, LSHM-CT-2004-503474.

\section{Results}

Findings from TpH-ir fiber density revealed significant (30-50\%) decrease, compared to baseline, both 3 days and 3 weeks after treatment in both hippocampus and the frontoparietal cortex. Results from ELISA showed that BDNF level in the frontoparietal cortex dropped significantly (30\%) 24 hours and 3 weeks after treatment. This decrease in BDNF concentration was also confirmed with 